\title{
On the Additional Symmetry; Many-Body Problem Related to the KP Hierarchy
}

\author{
Kazuhiro Hikami and Miki Wadati \\ Department of Physics, Faculty of Science, \\ University of Tokyo, \\ Hongo 7-3-1, Bunkyo-ku, Tokyo 113, Japan
}

(January 1994)

\begin{abstract}
Nonlinear integrable equations, such as the KdV equation, the Boussinesq equation and the KP equation, have the close relation with many-body problem. The solutions of such equations are the same as the restricted flows of the classical Calogero model, which is one-dimensional particle system with inverse square interactions. The KP hierarchy and the Calogero model share the same structure called "additional symmetry". This additional symmetry plays a crucial role in this relation.
\end{abstract}


In 1980s Sato school has developed the soliton theory and established the relationship with the infinite dimensional Lie algebra [1]. The basic equation is the KadomtsevPetviashvili (KP) hierarchy. Nonlinear integrable equations, such as the Korteweg-de Vries $(\mathrm{KdV})$ equation and the Boussinesq equation, can be considered as the reduction of the KP hierarchy.

Well known is that such nonlinear evolution equations are closely related not only to the string theory [2], but to the one-dimensional many-body problems [3,4]. The rational solutions of the equations correspond to the (restricted) flows of the so-called Calogero model [5]. For example, let us consider the KdV equation,

$$
u_{t}+6 u u_{x}+u_{x x x}=0 .
$$

The form of pole-expansion,

$$
u(x, t)=-2 \sum_{j=1}^{N} \frac{1}{\left(x-q_{j}(t)\right)^{2}},
$$

is the solution if $q_{j}$ follow the Hamiltonian flow induced by $\mathcal{I}_{3}$ (see below) with constraint,

$$
\sum_{j \neq k}^{N} \frac{1}{\left(q_{j}-q_{k}\right)^{3}}=0, \quad k=1,2, \cdots, N .
$$

The meaning of these relations still remains as an open problem [6].

In this letter we give the meaning based on the "additional symmetry". The additional symmetry of the KP hierarchy has been introduced in [7, 8]. This symmetry plays an important role in the matrix model for two-dimensional gravity, and provides a string theory, Virasoro constraint, and so on. On the other hand, the symmetry of the Calogero model has also been revealed for classical [9] and quantum [10] cases. We show that the Calogero model has the same structure with the KP hierarchy.

We begin with the Sato theory and consider the symmetry of the KP hierarchy. Let $\left\{t_{j}\right\}$ denote a set of independent variables. We set $x \equiv t_{1}$ and $\partial=\partial / \partial x$. The formulation is based on the Lax equations for the pseudo-differential operator, $L=\partial+u_{2} \partial^{-2}+u_{3} \partial^{-3}+\cdots$ :

$$
\frac{\partial L}{\partial t_{n}}=\left[B_{n}, L\right], \quad n=1,2, \cdots
$$


Here $u_{n}$ are the functions of $\left\{t_{j}\right\}$, and $B_{n}=\left(L^{n}\right)_{+}$is the truncation to the differential part of $L^{n}$. A negative power part of the differential operator $L^{n}$ will be written as $\left(L^{n}\right)_{-}$. The Lax equations (4) satisfy the zero-curvature equation, which is known as the Zakharov-Shabat equations,

$$
\frac{\partial B_{n}}{\partial t_{m}}-\frac{\partial B_{m}}{\partial t_{n}}+\left[B_{n}, B_{m}\right]=0, \quad n, m=1,2, \cdots
$$

Note that the Lax equations (4) give the integrability conditions of the linear system,

$$
\begin{aligned}
& L \psi(t, \lambda)=\lambda \psi(t, \lambda), \\
& \frac{\partial \psi(t, \lambda)}{\partial t_{n}}=B_{n} \psi(t, \lambda) .
\end{aligned}
$$

where $\psi(t, \lambda)$ is the Baker-Akhiezer function. This function can be written as

$$
\psi(t, \lambda)=W \exp \left(\sum_{n=1}^{\infty} t_{n} \lambda^{n}\right),
$$

where $W$ is the zero-th order pseudo-differential operator

$$
W=1+w_{1}(t) \partial^{-1}+w_{2}(t) \partial^{-2}+\cdots
$$

This dressing operator $W$ satisfies the Sato equation

$$
\begin{aligned}
& \frac{\partial W}{\partial t_{n}}=B_{n} W-W \partial^{n}, \\
& L W=W \partial .
\end{aligned}
$$

It is known that the KP hierarchy has the higher symmetry [7]. This symmetry plays an important role for the theory of the two-dimensional gravity [11]. We define the operator $M$ by

$$
M=W\left(\sum_{k=1}^{\infty} k t_{k} \partial^{k-1}\right) W^{-1}=W x W^{-1}+\sum_{k=2}^{\infty} k t_{k} L^{k-1} .
$$

This operator satisfies the Lax equation

$$
\frac{\partial M}{\partial t_{n}}=\left[B_{n}, M\right]
$$


It can be checked that the operators $L$ and $M$ are canonically conjugate,

$$
[L, M]=1
$$

By use of these operators we can construct the $W_{1+\infty}$ algebra [11],

$$
\left[\mathcal{W}_{n}^{(s)}, \mathcal{W}_{m}^{\left(s^{\prime}\right)}\right]=\left(\left(s^{\prime}-1\right) n-(s-1) m\right) \mathcal{W}_{n+m}^{\left(s+s^{\prime}-2\right)}+\cdots,
$$

with the $W$-operators, $\mathcal{W}_{n}^{(s)}=M^{n}(M L)^{s-1}$. The relation (12) and its generalized form,

$$
\left[L^{n}, \frac{1}{n} M L^{-n+1}\right]=1
$$

correspond to the string equation in the theory of the two-dimensional gravity [2]. Notice that (11) and (12) are consistent with the linear equation

$$
\frac{\partial \psi(t, \lambda)}{\partial \lambda}=M \psi(t, \lambda)
$$

For our purpose it is more convenient to rewrite (4), (11]) and (9) into

$$
\begin{aligned}
& \frac{\partial L}{\partial t_{n}}=\left[L,\left(L^{n}\right)_{-}\right], \\
& \frac{\partial M}{\partial t_{n}}=\left[M,\left(L^{n}\right)_{-}\right]+n L^{n-1}, \\
& \frac{\partial W}{\partial t_{n}}=-\left(L^{n}\right)_{-} W .
\end{aligned}
$$

Now we shall show that the $N$-body Calogero model, in which the particles interact through the pairwise inverse square potentials, share the same symmetry. The Hamiltonian of the Calogero model is

$$
\mathcal{H}=\sum_{j=1}^{N} p_{j}^{2}+\sum_{1 \leq j<k \leq N} \frac{2 a^{2}}{\left(q_{j}-q_{k}\right)^{2}} .
$$

The momentum $p_{j}$ and the position $q_{j}$ are canonical conjugate, that is, in terms of the Poisson bracket, $\left\{p_{j}, q_{k}\right\}=\delta_{j k}$. To discuss the symmetry, we use the classical $r$-matrix structure [12] for the $N \times N$ Lax matrix $\mathbf{L}$,

$$
\mathbf{L}=\sum_{j} p_{j} E_{j j}+\sum_{j \neq k} \frac{\mathrm{i} a}{q_{j}-q_{k}} E_{j k}
$$


Here $E_{j k}$ are the basic matrices, $\left(E_{j k}\right)_{l m}=\delta_{j l} \delta_{k m}$. The Lax matrix $\mathbf{L}$ satisfies the following identity,

$$
\{\mathbf{L} \stackrel{\otimes}{,} \mathbf{L}\}=\left[r_{12}, \mathbf{L} \otimes \mathbf{1}\right]-\left[r_{21}, \mathbf{1} \otimes \mathbf{L}\right] .
$$

Here $\{\stackrel{\otimes}{,}\}$ denotes the fundamental Poisson bracket [13], $\{\mathbf{A} \stackrel{\otimes}{,} \mathbf{B}\}=\sum\left\{A_{j k}, B_{l m}\right\} E_{j k} \otimes E_{l m}$. The classical $r$-matrix is defined by

$$
r_{12}=\sum_{j \neq k} \frac{-1}{q_{j}-q_{k}} E_{j k} \otimes E_{k j}+\sum_{j \neq k} \frac{1}{q_{j}-q_{k}} E_{j j} \otimes E_{k j} .
$$

Using the permutation operator, $\mathbf{P}=\sum_{j, k} E_{j k} \otimes E_{k j}$, we may express $r_{21}=\mathbf{P} r_{12} \mathbf{P}$. Remark that the classical $r$-matrix is slightly different from the one in [12]. The other intriguing structure is possessed by the diagonal matrix $\mathbf{Q}$,

$$
\mathbf{Q}=\operatorname{diag}\left(q_{1}, q_{2}, \cdots, q_{N}\right) .
$$

This matrix satisfies the following relation in terms of the classical $r$-matrix;

$$
\{\mathbf{Q} \stackrel{\otimes}{,} \mathbf{L}\}=\left[r_{12}, \mathbf{Q} \otimes \mathbf{1}\right]-\mathbf{P} .
$$

These equations lead to the following equations of motion;

$$
\begin{aligned}
\frac{\partial \mathbf{L}}{\partial t_{n}} & =\left[\mathbf{L}, \mathbf{M}_{n}\right], \\
\frac{\partial \mathbf{Q}}{\partial t_{n}} & =\left[\mathbf{Q}, \mathbf{M}_{n}\right]+n \mathbf{L}^{n-1} .
\end{aligned}
$$

Here $\mathbf{M}_{n}$ are $N \times N$ matrices defined by

$$
\mathbf{M}_{n}=n \operatorname{Tr}_{2}\left(r_{12} \cdot \mathbf{1} \otimes \mathbf{L}^{n-1}\right),
$$

where $\operatorname{Tr}_{2}$ means the trace of the matrices in the second space of a direct product. The time variable $t_{n}$ is associated with the flow for the conserved quantity $\mathcal{I}_{n} \equiv \operatorname{Tr} \mathbf{L}^{n}$, as

$$
\frac{\partial \mathbf{A}}{\partial t_{n}}=\left\{\mathcal{I}_{n}, \mathbf{A}\right\}
$$

One finds that the matrix $\mathbf{M}_{n}$ is equivalent to the results in [9]. 
One observes that (23) have appeared in the KP hierarchy. As the time $t_{n}$ denote the flow for the conserved quantities $\mathcal{I}_{n}$, only the finite quantities are independent for the $N$-body system. To compare the Calogero system with the KP hierarchy we must take the large $N$ limit, $N \rightarrow \infty$. The explicit construction can be seen from the matrix representation. We consider the following equation,

$$
\mathbf{Q}=\mathbf{U}\left(\sum_{k=1}^{\infty} k t_{k} \mathbf{H}^{k-1}\right) \mathbf{U}^{-1}
$$

Here we suppose that the matrix $\mathbf{H}$ does not depend on the "time" variables $t_{n}$. A simple calculation leads to (23) if we set

$$
\begin{aligned}
& \mathbf{M}_{n}=-\frac{\partial \mathbf{U}}{\partial t_{n}} \mathbf{U}^{-1}, \\
& \mathbf{L}=\mathbf{U H U}^{-1}
\end{aligned}
$$

These equations correspond to the Sato equation (9) in the KP hierarchy.

It is now straightforward to show the relationship between the KP hierarchy and the many-body problem from the generalized Lax equations and the additional symmetry. The $n$-th order KP equation corresponds to the flow for $\mathcal{I}_{n}$. Further the KdV hierarchy and the Boussinesq hierarchy, which are the reduction of the KP hierarchy, can be formulated. For example, the $\mathrm{KdV}$ hierarchy is the $n=2$ reduction of the KP hierarchy. We suppose the peudo-differential operator as $L^{2}=\partial^{2}+u$, or $\left(L^{2}\right)_{-}=0$. Then the $k$-th order $\mathrm{KdV}$ equation is obtained by $\partial L^{2} / \partial t_{2 k+1}=\left[L^{2},\left(L^{2 k+1}\right)_{-}\right]$, while the "even" time variables $t_{2 k}$ are suppressed, $\partial L^{2} / \partial t_{2 k}=0$. From the correspondence with the Calogero model, one concludes that the $k$-th order $\mathrm{KdV}$ equation is equivalent to the flow of $\mathcal{I}_{2 k+1}$ with the constraint $\operatorname{grad} \mathcal{I}_{2 k} \equiv 0$. These results are consistent with the suggestion in [3].

One of the author (KH) appreciates the Fellowship through the Japan Society for Promotion of Science. 


\section{REFERENCES}

[1] E. Date, M. Kashiwara, M. Jimbo and T. Miwa, in Proceedings of RIMS Symposium on Non-Linear Integrable Systems - Classical Theory and Quantum Theory, World Scientific, 1983 .

[2] E. Brézin and V. A. Kazakov, Phys. Lett. 236B, 144 (1990); M. R. Douglas and S. H. Shenker, Nucl. Phys. B335, 635 (1990); D. J. Gross and A. A. Migdal, Phys. Rev. Lett. 64, 127 (1990); M. R. Douglas, Phys. Lett. 238B, 176 (1990).

[3] H. Airaul, H. P. McKean and J. Moser, Comm. Pure Appl. Math. 30, 95 (1977); D. V. Choodnovsky and G. V. Choodnovsky, Nuovo Cimento 40B, 339 (1977); F. Calogero, Nuovo Cimento 43B, 177 (1978); I. M. Krichever, Funct. Anal. Appl. 12, 76 (1978).

[4] M. A. Olshanetski and A. M. Perelomov, Phys. Rep. 71, 313 (1981).

[5] F. Calogero, J. Math. Phys. 10, 2197 (1969); B. Sutherland, J. Math. Phys. 12, 246 (1971); J. Moser, Adv. Math. 16, 197 (1975).

[6] M. J. Ablowitz and P. A. Clarkson, Solitons, Nonlinear Evolution Equations and Inverse Scattering, Cambridge Univ. Press, 1991.

[7] A. Yu. Orlov and E. I. Shulman, Lett. Math. Phys. 12, 171 (1986).

[8] L. A. Dickey, Soliton Equations and Hamiltonian Systems, World Scientific, 1991.

[9] G. Barucchi and T. Regge, J. Math. Phys. 18, 1149 (1977); K. Hikami and M. Wadati, J. Phys. Soc. Jpn. 62, 3857 (1993).

[10] K. Hikami and M. Wadati, J. Phys. Soc. Jpn. 62, 4203 (1993); preprint (1994).

[11] A. Gerasimov, A. Marshakov, A. Mironov, A. Morozov and A. Orlov, Nucl. Phys. B357, 565 (1991); T. Yoneya, Comm. Math. Phys. 144, 623 (1992).

[12] J. Avan and M. Talon, Phys. Lett. 303B, 33 (1993); E. K. Sklyanin, preprint. 
[13] L. D. Faddeev and L. A. Takhtajan, Hamiltonian Methods in the Theory of Solitons, Springer, 1987. 